\title{
Whole-Face Approach with Hyaluronic Acid Fillers
}

This article was published in the following Dove Press journal:

Clinical, Cosmetic and Investigational Dermatology

\author{
Sylwia Lipko-Godlewska' \\ Željana Bolanča 2,3 \\ Lucie Kalinová ${ }^{4}$ \\ Irēna Kermen ${ }^{5}$ \\ Boris Onisak ${ }^{6}$ \\ Ildikó Papp ${ }^{7}$ \\ Margarita Rebrov ${ }^{8}$ \\ Greta Valančiené ${ }^{9}$ \\ 'Private Practice, Cracow, Poland; \\ 'University Clinical Hospital Center \\ "Sestre Milosrdnice", Zagreb, Croatia; \\ ${ }^{3}$ Private Clinic Milojević, Zagreb, Croatia; \\ ${ }^{4}$ Nemocnice Bulovka, Praha, Czech \\ Republic; ${ }^{5}$ Private Practice, Riga, Latvia; \\ ${ }^{6}$ Nobilis, Maribor, Slovenia; ${ }^{7}$ Koruti \\ Orvosesztetikai Centrum, Budapest, \\ Hungary; ${ }^{8}$ East-Tallinn Central Hospital, \\ Tallinn, Estonia; ${ }^{9}$ Private Practice, Kaunas, \\ Lithuania
}

Correspondence: Sylwia Lipko-Godlewska Private Practice, 30th Kasztelanska St., No. 2, Cracow, 30-II6, Poland

Tel +48 660483865

Email sgodlewska@gmail.com

\begin{abstract}
The use of hyaluronic acid fillers in aesthetic medicine has changed over the years and the procedure became one of the most common in the world. Understanding the ageing process of the face and the anatomical interrelationships in the face have dramatically influenced the use of the hyaluronic acid fillers and the assessment of the patient. It was supported by a new technology of products and by the delivery of tools (eg, blunt cannulas), face imaging, and innovative injection techniques. The whole-face approach challenges the practitioner to look at the face as a whole, and the patient to trust and rely on the treatment plan. Over the years, we have understood, that the most important outcome of aesthetic procedures is what does the whole face look like not static but in motion, and how do people read emotions from it. Nowadays, the result changes "the face information" and makes aesthetic procedures more satisfactory. In this manuscript, we reviewed essentials of the current treatment approach including patient's consultations, product selection and injection techniques used in different parts of the face. One size fits none; thus, we provided a general overview of hyaluronic acid fillers used in different indications and presented several treatment approaches to each region of the face.
\end{abstract}

Keywords: hyaluronic acid, whole-face approach, aesthetic medicine

\section{Introduction}

Hyaluronic acid (HA) fillers are the mainstay of the modern era of bioactive injectable materials for aesthetic purposes. The HA fillers injections are second most-frequently performed aesthetic medicine procedures in the world after botulinum toxin injections. ${ }^{1}$ The primary favourable characteristics that make the HA fillers popular are limited allergic reactivity, availability of an antidote, and a wide selection of products fulfilling different aesthetic goals. The main concepts of the use of the HA fillers include rejuvenation, beautification, and restoration. Rejuvenation aims to reverse ageingrelated changes in the skin, fat pads, bones and muscles by restoring the volume and the contour. Beautification procedures change innate proportions and ratios in the face to desired aesthetic ideals defined by a patient and a physician. Finally, restoration aims for the improvement of the skin quality.

Treatment with the HA fillers may influence the message conveyed by the face. In effect, patients may look less tired, less angry, less sad and, of course, less saggy. These changes trigger emotions for higher patient satisfaction.

The way how the HA fillers are used today has evolved in the last two decades. Data from early clinical trials shaped the early practice of the HA fillers use. In these clinical trials, single areas of the face were treated with relatively small amounts of the product. ${ }^{2-7}$ They enrolled patients with different severity of 
wrinkles, folds, deformity and volume deficits. Outcomes were focused on individual aspects, eg, improvement of wrinkles, folds and later augmentation. These studies created fundaments for product registration and evidencebased adaptation into clinical practice. They focused only on single goal, instead of on overall aesthetic effects, and none of them addressed the skin quality. Following clinical trial results published at the beginning of the current decade, traditional aesthetic treatments with the HA fillers focused on eliminating one or a few individual wrinkles instead of addressing the whole face. One of the first studies which aimed to change paradigm were published by Rzany et al 2012. ${ }^{8}$ The study enrolled patients seeking tissue augmentation for at least three of eight indications including periorbital lines, tear troughs, cheeks, cheek folds, nasolabial folds, upper lip lines, lips and marionette lines. Patients were treated with a range of five HA fillers; each designed to have physical properties specifically adapted to its indication. An improvement of all treated indications contributed to an overall improvement as rated both by the investigators and the participants. ${ }^{8}$ Authors underlined a difference between the proposed whole-face approach vs the traditional treatment. The quantity of the filler injected at baseline session was 2-3 times bigger than in a single-area oriented studies where the median quantity was below $2 \mathrm{~mL}^{2-7}$ In the earlier study of 10 female patients, Taub et al called this innovative approach a multi-syringe, and it describes well the limited scope of practice that was a few years ago. ${ }^{9}$ It is important to note that larger volumes of the HA fillers did not result in any safety compromise. The current trials, like the HARMONY study, investigated benefits of a rational, multimodal aesthetic treatment approach combining the use of the HA fillers and onabotulinumtoxinA, support versatile treatment planning including the use of different interventions. $^{10,11}$

The whole-face approach is a challenge; it needs to address patient's awareness and trust, product selection, and physician's skills in treatment planning and injection techniques. Here we present the best practices of the wholeface approach for aesthetic treatment with the HA fillers, which evolved over the last years of professional practice.

\section{Hyaluronic Acid Filler Product-Related Aspects}

With the vast array of the HA filler products currently available, the decision of which one to use in specific situations can be complicated and confusing. Product characteristics determine proper placement, product layering, and the possibility of use in different indications. All HA fillers consist of HA chains of various lengths. They differ significantly due to the manufacturing processes in properties such as concentration of HA, particle size and degree of cross-linking. These are required to achieve treatment goal and adapt to torsion, lateral shear, compression, and stretching arising from muscle movements and external forces in injected tissues.

Usually, the HA filler is described by the concentration expressed as mg of HA per mL. It is the total concentration of HA which in case of biphasic fillers may include both cross-linked and free HA. Injected HA is degraded by endogenous hyaluronidase. To increase resistance to degradation and modify biomechanical properties but maintaining biological activity, molecules of HA are cross-linked. Cross-linked HA fillers contain solid elastic and liquid viscous components. The $\mathrm{G}$ ' parameter (given in $\mathrm{Pa}$ ) describes the elastic properties of a product. The compression deforms purely elastic materials to a certain point. The HA fillers with higher $G$ ' resist dynamic forces from muscle movements and provide better support and lift, as well as longer duration of correction. The $G$ " is a measure of viscous properties of a filler, its flow properties when lateral shear is applied. The $\mathrm{G}^{*}$ comes from the sum of G' and G' and measures overall viscoelastic properties. ${ }^{12}$ Cohesivity describes the capacity of a filler not to dissociate, to maintain gel integrity necessary to support contours and diminish surface irregularities. The HA filler with lower $G^{\prime}$ and cohesivity tends to lose projection when the gel with high cohesivity resists compression and maintains the initial shape of deposit. ${ }^{13}$ Another property of the filler is its moldability, which allows correction of the shape of the implant after the injection.

Properties of fillers are essential for proper placement and the final effect of the treatment. Placing highly cohesive filler with high $G$ ' superficially makes the implant visible. Such fillers offer high lift and projection to the face and are useful for injecting deep subdermal layers or even periosteal, eg, when defining the new contour of a chin or a jawline. Low cohesivity filler does not have lifting properties and does not resist compressions quickly spreads through the tight connective tissue. Such fillers are mainly designed for injecting into superficial dermal layers to improve the skin quality and the hydration. Understanding biophysical characteristics of the HA filler allows rational selection of a product for a specific 
clinical application: rejuvenation, restoration or beautification. In the whole-face approach, one size fits none.

\section{Patient's Consultation}

The patient's perception of aesthetic medicine is full of emotions, trends, own perceptions or anxiety about seen artificial appearance, as a result of bad practice. Some patients may request the procedure or use of a specific product. It is important to listen to the patient, to try to understand what are patient thoughts or opinions and needs. The first consultation is the most crucial one in order to build trust that will result in a long-term relationship with the patient. Use of a mirror and photos, both static and in motion, helps communicate with the patient; it reveals to a patient the lateral aspect of the face. ${ }^{14}$ Individualized analysis includes assessing bones and fat pads volume loss, pattern of muscular contraction, and quality of the skin. Photographs before the treatment in static and motion create a benchmark for evaluation of the final effect.

During the first visit, patients need to get information about the difference between their wants and their real needs. This is when they should understand the "message" of their face (sadness, tiredness, anger, sagginess) followed by treatment aim, options, risks, and costs.

The treatment plan bases on sex, anatomy, facial harmony and individual adjacent structures, which are in the scope of beautification procedures; ageing and overall aesthetics are related to rejuvenation and restoration. All, patient's attitude, thoughts, opinion and the "message" of the face support the overall aim of treatment.

No part of the face expresses emotion independently. Each emotion involves all parts of the face, underlining the importance of the whole-face approach to facial aesthetics. Any treatment performed in one area will affect other regions. However, for some patients, discussion about feelings would be difficult; it is worth building the fundament of treatment on emotional anchor.

Start the procedures from these most beneficial for the patient. The first success will grow confidence to continue with the next steps. ${ }^{14}$ To introduce the whole-face approach, it is essential to understand how the volume loss in one area affect others instead of looking just at wrinkles and folds, which are typically indicated by patients as targets of the treatment. Typically, treatment with fillers starts in the mid-face, which contains essential hallmarks of facial beauty and is responsible for the youthful appearance. The mid-face is one of the first facial areas to show signs of ageing. The treatment of the cheek area attributes to an overall improvement in facial appearance. ${ }^{15}$ Treating one indication influences the other one, eg, treating the cheek affects the nasolabial fold, tear trough and infraorbital hollow; treating the temporal region influences the position of an eyebrow and eyelids.

\section{Emotions-Driven Treatment}

Dynamic and expressions of the face convey emotions. Some of them are wanted, and some of them are considered inappropriate. The former include happiness, attractiveness, rested and relaxed and other emotions associated with positive states of mind. The latter are the opposite of the former, eg, anger, being sad, unattractive, tired, stressed, sagged. These unpleasant expressions may develop with the onset of ageing and, typically, face ages more quickly than the patient's personality. Specific facial deficiencies are associated with negative emotional expressions. ${ }^{17-21}$ Faces may convey negative emotional messages, which do not result from the emotional states but from changes occurring in soft tissue, fat compartments, muscles, and bones. That is why physicians may not hear complaints about looking old, but about the effects of negative expressions. ${ }^{17}$ Treatment of unfavourable emotional attributes of the face is more challenging than addressing isolated areas.

Emotion-guided treatment is the latest step in the development of the whole-face approach concept. Since an expression of a single emotion involves many anatomical subunits of the face, treatment of a single deficiency, in isolation, may not satisfy the patient. Mauricio de Maio guided his treatment approach by following eight groups of negative and positive emotional cues. Negative emotional attributes contributed for looking tired, sad, angry and having a saggy appearance when positive were looking attractive, younger, more contoured, and either feminine for women or masculine for men. ${ }^{15}$ For example, tired appearance may involve saggy cheeks, depressions of temples, and dark circles or bags under eyes. Reducing unfavourable attributes and the strengthening of the positives need to treat all above areas of the face.

\section{Mid-Face}

Those specialists who limit themselves to meet patients' requirements may fail to prevent or combat ageing. The mid-face area between the eyebrows and the base of the nose contains relevant hallmarks of facial beauty and 
youthful appearance. The treatment of the mid-face is fundamental for the lifting effect, improvement of deficiencies, hollow appearance, and the nose shape.

\section{Cheeks}

With ageing, the upper cheek area flattens and changes in shape due to bone resorption and decreasing volume of fat pads. Deep cheek fat pads are crucial in midfacial rejuvenation, increase cheek volume and enhance malar projection. In most cases, volume replacement in lateral and mid cheeks is the starting point of the treatment. Depending on the extent of skin sagging, products with intermediate or high lifting capacity (intermediate or high G' and cohesivity), fair integrity and limited spreadability are used. Injection of the cheek with a single bolus will not provide optimal results and may lead to artificial appearance. ${ }^{16}$ The same is in case of the cheek overfilling, giving a bulging and unnatural result. Injections are deep to the bone or multilayer, including bone and also fat pad compartment.

The treatment of a cheek starts with an injection of two structural support points of the lateral midface: zygomatic arch and zygomatic prominence. ${ }^{16}$ The zygomatic arch supports a cheek, an eyebrow, and a lower eyelid, when the zygomatic prominence provides projection of the cheek and the shortening of the palpebral-malar sulcus. With established support, the next steps aim for the cheek volumization. Injection into the anteromedial cheek aims to improve medial lid-cheek junction, and it softens the tear trough. Deep direct injection into the infraorbital foramen, where the infraorbital artery originates can be dangerous. ${ }^{14,16}$ Thus, using a needle, protect the infraorbital foramen with an index finger or use a cannula to deliver a product to the anteromedial cheek. The next injection point of the lateral lower cheek addresses volume loss in the sunken area at the parotid level. This injection may also influence a jawline. Finally, treatment of the buccal area aims to correct the volume loss in the submalar region and prejowl sulcus.

The treatment of a cheek has a significant effect on an overall appearance and on neighbouring periorbital area, which may improve during the treatment of the cheeks, making additional treatment unnecessary. ${ }^{14}$ An attempt to improve the nasolabial crease or nasolabial fold during series of the cheek injections can be unsuccessful and lead to overfilling. Mowlds and Lambros have recently shown that volumizing the cheek did not improve the nasolabial fold. The patients with the filler injected into the nasojugal crease showed an improvement in the medial face. However, satisfactory improvement of the nasolabial fold needs a direct injection. ${ }^{17}$

\section{Periorbital Region}

The periorbital region creates fundaments of an attractive and beautiful look of the eyes. In the ageing process, the area shows the volume depletion of the soft tissue and the bone resorption of the orbit. In cases of infraorbital rim depression, the tear trough becomes visible as a shadow, sharp, distinct line or even an eye bag. The treatment plan is guided by the severity and etiology of the deformation (the atrophy of the skin and the subcutaneous fat, the orbital oculi muscle contraction or herniation of orbital fat or bone resorption). ${ }^{18,19}$

The tear trough was considered as the most challenging area to treat with HA. The tear troughs are different as underlying causes vary. ${ }^{18-21}$ Many techniques have evolved to address this cosmetic issue. ${ }^{22}$ Low G', G' and cohesivity HA fillers are safe to correct tear trough deformity. It will also decrease the risk of Tyndall effect resulting from a large quantity of HA filler in a thin-skinned area. ${ }^{14,22}$ Among the important vessels in the infraorbital region with pathways which must be identified and considered before injection are the infraorbital artery, the zygomaticofacial artery and angular vessels. However, because of significant anatomical variation in location, size, path and branching patterns of facial arteries or veins, ${ }^{23,24}$ we advice routine aspiration before injection of every area to avoid unintentional and serious complications. The upper third of the face is most susceptible to vascular complications.

In general, an injection is along or below the orbital rim, below the defect. The injection technique depends on the clinical picture. Patients with shadows can be injected subdermally with a cannula or at the periosteal level with a cannula or a needle using micro-deposits of HA. A combination of both approaches is beneficial for patients with mild to moderate lines. Recently, Bagci has proposed a new technique for the correction of the tear trough deformity derived from malar fat reduction and changes of skin quality by injecting the HA filler into the preseptal space. ${ }^{25}$ The advantages are limited migration of the filler captured in the preseptal space and a smaller amount of the material used. If the deformity extends to the nasojugal groove, treatment requires a combination of subdermal and periosteal approaches. Volumization can be performed with either needles or blunt cannulas injecting multiple 
microdeposits, followed by molding of visible irregularities.

Fullness of the upper lid and the supraorbital brow define attractive periorbital complex; the tail of the brow is higher than the medial end; the lateral canthus has a smooth contour with no bony rim and its position is $5^{\circ}$ higher than the medial canthus. ${ }^{26}$ The lateral canthal region, the temple and the eyebrow need a precise treatment with medium $G^{*}$ filler to avoid floating of these areas. Shaping of the eyebrow consists of micro boluses injected subdermally or as periosteal deposits. Treatment of the lateral canthal region needs vertical puncture and deposition of small boluses using the retrograde technique.

\section{Nose}

The HA fillers injections are an excellent alternative for rhinoplasty. The use of HA is less invasive, less costly and requires shorter recovery time than a surgery. The area is reserved for advanced injectors only. The treatment combines injections aiming for beautification (correction of asymmetry) and rejuvenation since the shape of the nose changes with ageing.

Adequate knowledge of the proper techniques and patient's anatomy is essential for the nasal filling. When using a needle, injections are placed directly on the perichondrium and periosteum to avoid the main vessels present in the upper fatty and fibromuscular layers and minimalize the vascular compromise risk. Very low, flat noses and those with an excessive hump need the use of a needle rather than a cannula. ${ }^{14,27}$ The minimal approach includes injection of HA directly into the tip of the nose, which drops with age and due to facial animation. ${ }^{28}$ The volume of a filler varies on the size of the nose, skin thickness, and a number of sites treated. ${ }^{14}$ Single bolus should be up to $0.1 \mathrm{~mL}$ or less, and subsequent volume should be injected using the linear retrograde technique. Fillers with high $\mathrm{G}^{*}$ and cohesivity, provide sustained projection, limited spreadability and swelling optimal for use in the area.

Patients with deep nasolabial angle require its opening with the filling of the anterior nasal spine. A deep injection expands the inferior part of the membranous septum, increasing the angle to $\geq 90^{\circ}$ depending on the desired aesthetic endpoint dependent on the patient and anatomy. In patients with flat nostrils the medial crura should be expanded. Moldability of a filler can help to create and maintain a desirable shape (eg, a teardrop shape). Using the HA fillers, columellar retraction can be corrected by injections into the membranous septum. Too small columella can be filled, but its basis can not be too wide, what requires control of a shape with fingers. ${ }^{14,29}$

Excessive nasal hump or underprojected nasal dorsum increases the nasofrontal angle. Filling the concavity makes the dorsum straighter without surgical hump removal. The injection is close to the periosteum into the muscular and subdermal layers. Injections onto the bony and cartilaginous dorsum increase the nose projection on the profile view and narrow the nose on the frontal view. An injection into the cartilaginous dorsum smoothes the junction between the tip and the dorsum. ${ }^{14}$

In 2016, Respaldo introduced a nasal injection technique called the Eiffel Tower Nose-Lift. ${ }^{30}$ It consists of series of medial injections allowing avoidance of the main vessels. It bases on creating a tip support in the nasal spine, forming of the columellar strut, followed by shaping the tip and the correction of the nasal dorsum. ${ }^{30}$

\section{Lower-Face and Lips}

Treatment areas of the lower face include a chin, jaws and lips. With ageing, in the highly animated perioral zone, tissue firmness decreases faster than the strength of mimetic muscles, creating an imbalance between the tissue resistance and muscle domination. ${ }^{26}$ The HA filler deposits brace the perioral tissue resistance. Jaw enhancements with fillers augment and sharpen the jaw, by adding volume and contour lost during changes of soft-tissue (excessive fat or skin laxity) and bone resorption. Thus, the treatment of a chin and a jawline requires the use of fillers restoring/creating facial volume with different lifting properties. ${ }^{31,32}$ It is common to combine the treatment of the chin and the jawline.

\section{Chin}

The chin maintains the balance of the lower part with the overall facial aesthetics and its beautification, rejuvenation, and restoration offer dramatic improvement. ${ }^{33}$ However, it belongs to the areas that are mostly neglected by patients and most often its treatment needs to be encouraged by professionals. An assessment of the chin includes evaluation of occlusion, skeletal and dental relationships, lateral fullness, presence of the marionette lines, and the chin projection along the nasal vertical line. Both profile and anterior views are critical for the final effect. Preparation to the treatment includes an assessment of the platysma for the presence of the necklines, active contraction when speaking, evaluation of the ratio between the 
distance from oral commissure and the chin to the height of the chin, which ideally is $1: 1 .^{34}$

The HA fillers are the treatment of choice for defining a new contour of a chin or a jawline. Since the lower face is highly mobile, the ideal filler has intermediate to the highest possible $G$ ' and cohesivity, offering very high integrity, lift, and contouring properties. Dense fillers are a durable alternative to surgical treatments in sculpting, shaping, and contouring in the chin and jaw area. ${ }^{31,32}$

An injection into mental crease is an integral part of the beautification process. It allows regulating the mentioned ratio between the area below a lower lip and a chin, by opening the labiodental angle and elongation of the chin. Patients with soft-tissue volume loss, reduced skin elasticity or bone resorption in the mandibular area would benefit from the rejuvenation of the mental crease. This is also a region for myomodulation. All depressors (more active while we get older) can be pressed by the injected HA filler. Dr M. de Maio described this area as C1. There are two fanning, subcutaneous, retrograde injections per side. Localization of the sublabial vessels needs attention during the injections. ${ }^{34,35}$

The periosteal bolus into the chin apex may correct undesirably recessed chin increasing its anterior projection and rounding. Two injections in the most anterior midpoint of the chin improve its anterior projection. ${ }^{34}$ All points, the mental crease, chin apex, and anterior chin can be reached with blunt cannula using one lateral entry point. ${ }^{35}$ They form the basis of a tapered shape of the chin. An additional injection into the most inferior midpoint contour of the chin in the submental area additionally enhances the effects of injection into the chin apex and the anterior chin. ${ }^{36}$ Periosteal injections of small boluses into the lateral lower chin may be desired to create a wider, squareshaped chin, especially in male patients.

Ageing-related soft tissue atrophy lateral to the anterior chin results in a triangle-shaped prejowl sulcus. ${ }^{14,36}$ A deep subcutaneous fanning ensures filling of the prejowl sulcus. Aspiration is necessary and the mental vessels are present close to the injected area. An injection performed from a lateral entry deposes HA in the distal parts of the prejowl triangle. ${ }^{32}$ Progression of fat and soft-tissue atrophy and muscular hyperactivity lead to the development of marionette lines and a sad, saggy appearance. The filler selection bases of the depth of the lines and wrinkles and involves two injections per side. ${ }^{14}$ The first one delivers the filler subcutaneously and medially to the marionette line with most of the volume to the top third of it. A lateral injection may increase the visibility of the fold. The second injection is made in the modiolus using a smaller volume than the first one and supports the oral commissure. Very deep folds may require multilevel injections (deep and superficial ones). ${ }^{14,32}$ Increased caution is needed because of the presence of inferior labial and sublabial arteries and veins.

\section{Jawline}

The perfect jawline is well defined and smooth from the angle of the jaw until the chin, uninterrupted by sulci or saggy skin. In contrast to the mid-face, the jawline demonstrates sharp transitions and contours. Ageing processes and the impact of environmental exposures lead to decreased muscle and bone mass in the jawline. Treatment strategies aim for mandible angle enhancement and/or contouring of the jawline. In effect, it is possible to achieve a more prominent outline of the face and to shape the neck, too. ${ }^{14,35}$

Patients with good chin projection but lack of definition of the jaw are the ideal candidates for rejuvenation with the HA fillers injections in this area. ${ }^{14}$ Contour a sharp jawline requires highly viscous fillers with high-lifting properties. Fillers can be placed along the mandible body using retrograde fanning with a needle or a cannula. ${ }^{14,35}$ Next, fullness of the jawline should be followed by enhancement of mandibular angle to promote a posterior jawline contour. From the entry above the midline of the mandibular angle perform few retrograde threads of the filler. ${ }^{14,35}$ The effect of the jawline contouring and mandibular angle augmentation largely depends on the amount of the product used, however, too low doses would not be sufficient.

\section{Lips}

HA fillers are highly suitable for the lip beautification and augmentation. Treatment aims for the attractiveness and youthfulness of the perioral area. Age-dependent volume loss leads to its flattering, shrinking, drying and fading into the facial plane. In the lip, individual anatomical assessment and specific treatment goals dictate the choice of the filler. In patients who do not desire augmentation or changes in the shape of the lips, use the filler with the lowest $G$ ' and cohesivity adapting to the shape of the tissue, to achieve improvement of the skin quality, its hydration, smoothness, and elasticity. ${ }^{35}$ Such a strategy would help maintain the feminine look of older women who are afraid of excessive lip augmentation. Low-volume superficial injections are effective in gaining more radiant age-appropriate appearance after rejuvenation. ${ }^{37}$ Patients 
expecting beautification or beautification and rejuvenation need the filler with intermediate $G^{\prime}$ and viscosity to augment the size and shape of the lips. ${ }^{35}$

There is no single standard of the ideal lips. Giving the lips the proper size, contour, volume, and projection is a challenge. Lips attract attention and do not forgive imperfections. ${ }^{14}$ The era of overfilled lips that started in the '00s has passed. Today the main aim is to achieve a pleasing, natural appearance of harmony between lips, chin, nose, and the contour of a jawline. ${ }^{38}$ The ratio between the upper and the lower lips is between 1:1 to $1: 2$, and at the profile view of the upper and lower lip should be about $3.5 \mathrm{~mm}$ and $2.2 \mathrm{~mm}$ forward, respectively. However, it depends on patients' preferences, ie, some patients prefer more abundant and projected lower lip.

An assessment includes an evaluation of the volume, contour, and shape of the lips. Patients require dental evaluation since the height of teeth and occlusion affect lip projection. Lip volume can be increased with vermillion injections using a blunt cannula. Injections into the Cupid's bow and borders of the lips (the frame of the lips) enhance the contour of the lips. The border of the lip should not be straight; filling needs to vary continuously across the lip to pronounce its natural shape. ${ }^{19}$ Vermillion overcorrection eliminates the Cupid's bow and creates socalled "duck lip". 14,19,38 The shape and projection of the lips can be corrected in some patients by injecting small bolus into the mucosa of the medial tubercle of the upper lip and the lateral tubercles of the lower lip.

Elderly patients may benefit from minimal injections of the philtral columns. Redefining of philtrum needs to preserve its natural shape, narrow near the nose and wider at the connection with the Cupid's bow. Injections at the lateral aspect of the lower lip supporting the ascendant line correct oral commissures. The final step is to correct the perioral wrinkles by micro aliquots of HA injected subdermally avoiding the overcorrection flattening the area.

\section{Upper-Face}

A female's temple should be flat without any depressions or hollowings. The ideal forehead has only few or no lines and a gentle $\left(12-14^{\circ}\right)$ convex curve. Women eyebrows should be well demarcated and arched with the highest point at the level of the lateral limbus of the eye. Male eyebrows are also well defined but horizontal. Soft tissue volume loss and bone remodelling lead to an increased protrusion of the upper forehead, flattening of the lower forehead and eyebrows, increase in glabellar height and width and temporal hollowing. ${ }^{26}$
Botulinum toxin serotype A (BoNT-A) is the treatment of choice for the upper-face in patients with dynamic wrinkles. However, dynamic lines are likely to improve with BoNT-A treatment, but deep and static wrinkle improvement is limited. HA fillers, other interventions or combination treatments can be offered in such cases. Typically, the HA filler injections should follow BoNT-A injections about two weeks later. $^{39}$ Delay is required since the BoNT-A effect is not immediate; ${ }^{37}$ however, combining both treatments at one visit is not contraindicated. The main reason for HA fillers use in the upper face is optimising the volume and tissue support. Treatment strategies include a temple volumization, an eyebrow shaping, and a forehead contouring.

\section{Temples}

Patients do not frequently request the treatment of the temples. The treatment of other regions of the face may increase disproportion between general facial fullness and hollow temples. $^{14}$

Depending on the morphology of temple depression treatment would include single bolus deep down to the bone or retrograde subcutaneous injection. The HA filler volume varies from 0.5 to $1 \mathrm{~mL}$ per side in mild/moderate and severe cases, respectively. ${ }^{14}$ Aspiration is mandatory due to the presence of the superficial and deep temporal vessels.

\section{Forehead and Glabella}

Dynamic lines of glabella and the forehead are commonly treated with BoNT-A. Both static horizontal lines and the contour of the forehead may require treatment with the HA fillers. It includes periosteal injections of a product with medium viscosity; typically a few deep small boluses across the forehead followed by gentle molding. Endpoint evaluation needs assessment of the profile view. ${ }^{14,19,40,41}$

In the glabella, injections used to treat static lines must be very superficial and small (micro-aliquots). ${ }^{42}$ An injection with a cannula in the midline at the supraperiosteal level would be required to reshape the glabella. The glabella was the most common treatment site leading to blindness due to vascular complications with injectable fillers. ${ }^{43}$

\section{Eyebrows}

The HA fillers are used to shape, augment, and contour the eyebrow. The female eyebrow should be over the supraorbital margin, and its head should be lower than the tail; the male eyebrow lies on the supraorbital margin and is lower than in female patients. Asymmetry of the eyebrows is a common problem that can be addressed by beautification procedures 
using the HA fillers. The contraction of muscles in the glabellar complex and the orbicularis oculi muscle affects the shape and the position of the eyebrows. Treatment with BoNT-A abolishes the activity of these muscles and changes the position of the eyebrows. If the treatment effect is insufficient, the reason behind the unwanted shape and position of the eyebrows is loss of volume. ${ }^{41}$

A small aliquot of HA filler injected subcutaneously into the lateral part of the eyebrow elevates the tail of the eyebrow. ${ }^{40}$ However, temporal volume replacement would give a similar effect. ${ }^{14}$ Periosteal injection elevates the tail of the eyebrow when subdermal to change an eyebrow contour. Typically, attempts of subperiosteal injections fail. ${ }^{41}$ Injections into the central part of the eyebrow and its head contour and elevate these parts and are subdermal. The injections remain at or are superior to the superior orbital rim, but not below the rim. ${ }^{40}$ Decreasing the volume injected in each point, starting from the tail to the head of the eyebrow is essential for their final shape and position. ${ }^{14,43}$

\section{Summary}

The HA fillers revolutionized the fillers market thanks to their properties and possibility of use in different indications. Relatively easy treatment focused on isolated wrinkles and folds evolved with beautification and rejuvenation procedures combining contouring, augmentation, and shaping different face regions. We like to structure everything. By dividing the face into three areas and evaluating individual features of the face in isolation, the outcome is perceived as the overall aesthetic collective interplay. ${ }^{44}$

The whole-face approach needs building trust between the patient and the professional and requires joint planning. Emotional anchor supports collaboration and shaping of the new face "message". The outcome needs a stepwise execution of the plan, ensuring that all goals are achieved with the best allocation of resources. This article provided a general overview of how the HA filler may be used in different applications and presented several treatment approaches to each region of the face. Understanding the importance of patient's assessment, practicalities, safety precautions, and injection techniques of different treatment approaches help to create individual treatment plans to meet the patient's expectations.

\section{Acknowledgments}

Writing and editorial assistance was provided to the authors by Marcin Balcerzak of Medink, Poland and funded by Allergan CZ s.r.o., Praha, Czech Republic. This manuscript was prepared based on the discussion of experts on the meetings organized by Allergan. All authors met the ICMJE authorship criteria. Neither honoraria nor payments were made for the authorship. Allergan provided suggestions for topic ideas and for authors of this manuscript to Medink Marcin Balcerzak, Poland. Allergan was not involved in the development of the manuscript neither with the authors nor with the vendor. Allergan had an opportunity to review the final version of the manuscript and provide comments, however, the authors maintained complete control over the content of the paper.

\section{Disclosure}

The authors report no conflicts of interest in this work.

\section{References}

1. International Society of Aesthetic Plastic Surgeons. IAPAS international survey on aesthetic/cosmetic procedures in 2017; 2018. Available from: www.isaps.org. Accessed July 29, 2019.

2. Baumann LS, Shamban AT, Lupo MP, et al. Comparison of smooth-gel hyaluronic acid dermal fillers with cross-linked bovine collagen: a multicenter, double-masked, randomized, within-subject study. Dermatol Surg. 2007;33:S128-S135. doi:10.1111/j.1524-4725.2007.33352.x

3. Callan P, Goodman GJ, Carlisle I, et al. Efficacy and safety of a hyaluronic acid filler in subjects treated for correction of midface volume deficiency: a 24 month study. Clin Cosmet Investig Dermatol. 2013;6:81-89. doi:10.2147/CCID.S40581

4. Eccleston D, Murphy DK. Juvéderm $\left({ }^{\circledR}\right)$ Volbella ${ }^{\mathrm{TM}}$ in the perioral area: a 12-month prospective, multicenter, open-label study. Clin Cosmet Investig Dermatol. 2012;5:167-172. doi:10.2147/CCID.S35800

5. Narins R, Coleman WP, Donofrio LM, et al. Improvement in nasolabial folds with a hyaluronic acid filler using a cohesive polydensified matrix technology: results from an 18-month open-label extension trial. Dermatol Surg. 2010;36:1800-1808. doi:10.1111/ j.1524-4725.2010.01735.x

6. Nast A, Reytan N, Hartmann V, et al. Efficacy and durability of two hyaluronic acid-based fillers in the correction of nasolabial folds: results of a prospective, randomized, double-blind, actively controlled clinical pilot study. Dermatol Surg. 2011;37:768-775. doi:10.1111/j.1524-4725.2011.01993.x

7. Rzany B, Cartier H, Kestermont P, et al. Correction of tear troughs and periorbital lines with a range of customized hyaluronic acid fillers. J Drugs Dermatol. 2012;11(1 Suppl):s27-s34.

8. Rzany B, Cartier H, Kestemont P, et al. Full-face rejuvenation using a range of hyaluronic acid fillers: efficacy, safety, and patient satisfaction over 6 months. Dermatol Surg. 2012;38:1153-1161. doi:10.1111/ j.1524-4725.2012.02470.x

9. Taub AF, Sarnoff D, Gold M, Jacob C. Effect of multisyringe hyaluronic acid facial rejuvenation on perceived age. Dermatol Surg. 2010;36:322-328. doi:10.1111/j.1524-4725.2009.01436.x

10. Narurkar VA, Cohen JL, Dayan S, et al. A comprehensive approach to multimodal facial aesthetic treatment: injection techniques and treatment characteristics from the HARMONY study. Dermatol Surg. 2016;42:S177-S1791. doi:10.1097/DSS.0000000000000743

11. Weinkle SH, Werschler WP, Teller CF, et al. Impact of comprehensive, minimally invasive, multimodal aesthetic treatment on satisfaction with facial appearance: the HARMONY study. Aesthet Surg J. 2018;38:540-556. doi:10.1093/asj/sjx179

12. Kablik J, Monheit GD, Yu L, Chang G, Gershkovich J. Comparative physical properties of hyaluronic acid dermal fillers. Dermatol Surg. 2009;35:302-312. doi:10.1111/j.1524-4725.2008.01046.x 
13. Sundaram H, Rohrich RJ, Liew S, et al. Cohesivity of hyaluronic acid fillers: development and clinical implications of a novel assay, pilot validation with a five-point grading scale, and evaluation of six U.S. Food and Drug Administration-approved fillers. Plast Reconstr Surg. 2015;136:678-686. doi:10.1097/PRS.0000000000001638

14. de Maio M, Rzany B. Injectable Fillers in Aesthetic Medicine. 2nd ed. Heidelberg: Springer-Verlag; 2014.

15. Glaser DA, Kenkel JM, Paradkar-Mitragotri D, Murphy DK, Romagnano L, Drinkwater A. Duration of effect by injection volume and facial subregion for a volumizing hyaluronic acid filler in treating midface volume deficit. Dermatol Surg. 2015;41:942-949. doi:10.1097/DSS.0000000000000416

16. Cotofana S, Schenck TL, Trevidic P, et al. Midface: clinical anatomy and regional approaches with injectable fillers. Plast Reconstr Surg. 2015;136:219S-234S. doi:10.1097/PRS.0000000000001837

17. Mowlds DS, Lambros V. Cheek volumization and the nasolabial fold. Plast Reconstr Surg. 2018;141:1124-1129. doi:10.1097/ PRS.0000000000004341

18. Lee JH, Hong G. Definitions of groove and hollowness of the infraorbital region and clinical treatment using soft-tissue filler. Arch Plast Surg. 2018;45:214-221. doi:10.5999/aps.2017.01193

19. Turkmani MG. New classification system for tear trough deformity. Dermatol Surg. 2017;43:836-840. doi:10.1097/DSS.0000000000001056

20. Bass LS. Injectable filler techniques for facial rejuvenation, volumization, and augmentation. Facial Plast Surg Clin North Am. 2015;23:479-488. doi:10.1016/j.fsc.2015.07.004

21. Matarasso SL, Carruthers J, Jewell ML, et al. Consensus recommendation for soft-tissue augmentation with nonanimal stabilized hyaluronic acid (Restylane). Plast Reconstr Surg. 2006;117:3S-34S. doi:10.1097/01.prs.0000204759.76865.39

22. Sharad J. Dermal fillers for the treatment of tear trough deformity: a review of anatomy, treatment techniques, and their outcomes. J Cutan Aesthet Surg. 2012;5:229-238. doi:10.4103/09742077.104910

23. Palomar-Gallego MA, Gómez-Esquer F, Gómez-Sánchez SM, DíazGil G, Linares García-Valdecasas R. Influence of the topographic vascular distribution of the face on dermal filler accidents. Dermatology. 2019;235:156-163. doi:10.1159/000495292

24. Koziej M, Trybus M, Hołda M, et al. Anatomical map of the facial artery for facial reconstruction and aesthetic procedures. Aesthet Surg J. 2019;39(11):1151-1162. doi:10.1093/asj/sjz028

25. Bagci B, New A. Technique for the correction of tear trough deformity via filler injections. Plast Reconstr Surg Glob Open. 2018;6: e1901. doi:10.1097/GOX.0000000000001901

26. Swift A, Remington BK. The mathepatic of facial beauty. In: Johes DH, Swift A, editors. Injectable Fillers: Facial Shaping and Contouring. Oxford: Wiley-Blackwell; 2019:29-61.

27. Jung GS. Filler rhinoplasty based on anatomy: the dual plane technique. JPRAS Open. 2019;20:94-100. doi:10.1016/j.jpra.20 19.04.002

28. de Maio M. The minimal approach: an innovation in facial cosmetic procedures. Aesthetic Plast Surg. 2004;28:295-300. doi:10.1007/ s00266-004-0037-1

29. Lee YI, Yang HM, Pyeon HJ, Lee HK, Kim HJ. Anatomical and histological study of the arterial distribution in the columellar area, and the clinical implications. Surg Radiol Anat. 2014;36:669-674. doi:10.1007/s00276-013-1253-7
30. Raspaldo H. Eiffel tower nose-lift: anatomical basis and concepts for safe and effective nasal injections. Plast Reconstr Surg Glob Open. 2016;4:e1167. doi:10.1097/GOX.0000000000001167

31. Ogilvie P, Sattler G, Gaymans F, et al. Safe, effective chin and jaw restoration with VYC-25L hyaluronic acid injectable gel. Dermatol Surg. 2019;45:1294-1303. doi:10.1097/DSS.0000000000001960

32. Ogilvie P, Benouaiche L, Philipp-Dormston WG, et al. VYC-25L hyaluronic acid injectable gel is safe and effective for long-term restoration and creation of volume of the lower face. Aesthet Surg J. 2020;1-12.

33. Romo T, Lanson BG. Chin augmentation. Facial Plast Surg Clin North Am. 2008;16:69-77. doi:10.1016/j.fsc.2007.10.001

34. de Maio M, Wu WTL, Goodman GJ, Monheit G. Facial assessment and injection guide for botulinum toxin and injectable hyaluronic acid fillers: focus on the lower face. Plast Reconstr Surg. 2017;140:393e-404e. doi:10.1097/PRS.0000000000003646

35. Braz A, Humphrey S, Weinkle S, et al. Lower face: clinical anatomy and regional approaches with injectable fillers. Plast Reconstr Surg. 2015;136:235S-257S. doi:10.1097/PRS.0000000000001836

36. Vedamurthy M. Dermal fillers in lower face rejuvenation. In: Sharad J, Vedamurthy M, editors. Aesthetic dermatology: Current Perspectives. Daryaganj: Jaypee Brothers Medical Publishers; 2019:218-222.

37. Hilton S, Sattler G, Berg AK, Samuelson U, Wong C. Randomized, evaluator-blinded study comparing safety and effect of two hyaluronic acid gels for lips enhancement. Dermatol Surg. 2018;44:261-269. doi:10.1097/DSS.0000000000001282

38. Humphrey S. The Lips. In: Johes DH, Swift A, editors. Injectable Fillers: Facial Shaping and Contouring. Oxford: Wiley-Blackwell; 2019:149-164.

39. Carruthers A, Carruthers J, Monheit GD, Davis PG, Tardie G. Multicenter, randomized, parallel-group study of the safety and effectiveness of onabotulinumtoxinA and hyaluronic acid dermal fillers (24-mg/mL smooth, cohesive gel) alone and in combination for lower facial rejuvenation. Dermatol Surg. 2010;36:2121-2134. doi:10.1111/ j.1524-4725.2010.01705.x

40. Sykes JM, Cotofana S, Trevidic P, et al. Upper face: clinical anatomy and regional approaches with injectable fillers. Plast Reconstr Surg. 2015;136:204S-218S. doi:10.1097/PRS.0000000000001830

41. de Maio M, Swift A, Signorini M, Fagien S. Facial assessment and injection guide for botulinum toxin and injectable hyaluronic acid fillers: focus on the upper face. Plast Reconstr Surg. 2017;140:265e276e. doi:10.1097/PRS.0000000000003544

42. Carruthers J, Carruthers A. A prospective, randomized, parallel group study analyzing the effect of BTX-A (Botox) and nonanimal sourced hyaluronic acid (NASHA, Restylane) in combination compared with NASHA (Restylane) alone in severe glabellar rhytides in adult female subjects: treatment of severe glabellar rhytides with a hyaluronic acid derivative compared with the derivative and BTX-A. Dermatol Surg. 2003;29:802-809. doi:10.1046/j.15244725.2003.29212.x

43. Pavicic T, Yankova M, Schenck TL, et al. Subperiosteal injections during facial soft tissue filler injections-Is it possible? J Cosmet Dermatol. 2020;19(3):590-595. doi:10.1111/jocd.13073

44. Beleznay K, Carruthers JD, Humphrey S, Jones D. Avoiding and treating blindness from fillers: a review of the world literature. Dermatol Surg. 2015;41:1097-1117. doi:10.1097/DSS.000000 0000000486 


\section{Publish your work in this journal}

Clinical, Cosmetic and Investigational Dermatology is an international, peer-reviewed, open access, online journal that focuses on the latest clinical and experimental research in all aspects of skin disease and cosmetic interventions. This journal is indexed on CAS.
The manuscript management system is completely online and includes a very quick and fair peer-review system, which is all easy to use. Visit http://www.dovepress.com/testimonials.php to read real quotes from published authors.

Submit your manuscript here: https://www.dovepress.com/clinical-cosmetic-and-investigational-dermatology-journal 